\title{
La estimulación mecánica aferente no reduce sistemáticamente el temblor de pacientes con temblor esencial
}

\author{
Lora-Millán, Julio Salvador ${ }^{a^{*}}$; López-Blanco, Roberto ${ }^{\mathrm{b}}$; Gallego, Juan Álvaro ${ }^{\mathrm{a}}$; \\ González de la Aleja, Jesús ${ }^{b}$; Rocon, Eduardo ${ }^{a}$ \\ ${ }^{a}$ Centro de automática y robótica (CAR), CSIC-UPM, Madrid, España, \\ 'Instituto de investigación sanitaria Hospital 12 de Octubre, Madrid, España. \\ *Autor al que dirigir correspondencia: julio.lora@csic.es
}

\begin{abstract}
In spite of being one of the major causes of disability, Essential Tremor (ET) is not effectively handled in 50\% of cases. We have hypothesised that tremor in ET could be reduced by recruiting afferent pathways through mechanical stimulation. We have designed a platform to stimulate the forearm and hand of the patient with piezoelectric actuators while the tremor is measured using inertial sensors. We have studied the effect of four different stimulation strategies in 18 ET patients, each stimulation strategy was characterized by the frequency of the vibration. Although the response to stimulation was very heterogeneous across patients and strategies, the majority of patients increased their tremor (between 50\% and 72\%, depending on the strategy), while a very reduced proportion (between 5 and 22\%) of patients decreased their tremor amplitude. However, we considered that these results were difficult to explain because the high variability of the tremor that we have assessed: during equally long trials without stimulation, tremor increased significantly in $45 \%$ of patients and decreased in $22 \%$ of them. Therefore, we claim that mechanical vibration does not have a consistent and homogenous effect on ET tremor.
\end{abstract}

Keywords: Essential tremor, mechanical stimulation, piezoelectric actuators, inertial sensors.

\footnotetext{
Resumen

A pesar de ser una de las principales causas de discapacidad, el temblor esencial (TE) no se consigue tratar de manera efectiva en el $50 \%$ de los casos diagnosticados. Nosotros partimos de la hipótesis de que, al estimular las vías aferentes a través de vibración mecánica, el temblor en pacientes con TE podría reducirse. Para comprobarlo, se ha desarrollado una plataforma para estimular el antebrazo y la mano del paciente usando actuadores piezoeléctricos mientras se mide el temblor utilizando sensores inerciales.
} 
La estimulación mecánica aferente no reduce sistemáticamente el temblor de pacientes con temblor esencial

Después de realizar ensayos con 18 sujetos, se ha analizado el efecto de aplicar cuatro estrategias de estimulación a diferente frecuencia de vibración. Aunque la respuesta a la estimulación fue muy heterogénea teniendo en cuenta los pacientes y las estrategias aplicadas, en la mayoría de los pacientes el temblor aumentó significativamente (entre 50\% y 72\%, según la estrategia), mientras que para una proporción reducida de pacientes (entre 5 y 22\%) disminuyó la amplitud de su temblor. No obstante, se considera que estos resultados son dificiles de explicar debido a la gran variabilidad del temblor: durante ensayos de igual duración, pero sin aplicar estimulación, el temblor aumentó significativamente en el $45 \%$ de los pacientes y disminuyó en el $22 \%$ de ellos. Por lo tanto, podemos afirmar que la vibración mecánica no tiene un efecto consistente y homogéneo en el temblor esencial.

Palabras clave: Temblor esencial, estimulación mecánica aferente, actuadores piezoeléctricos, sensores inerciales

\section{Introducción}

El temblor esencial (TE) es uno de los trastornos del movimiento más prevalentes en adultos afectando aproximadamente al 5\% de las personas mayores de 65 años (Louis 2005). El TE se manifiesta como un temblor postural o cinético bilateral, en gran parte simétrico, que afecta a las manos y los antebrazos, y suele ir acompañado de temblor en la cabeza (Deuschl 1998). Sin embargo, el temblor solo se controla de manera efectiva en el 50\% de todos los pacientes (Deuschl 2011), por lo que existe la necesidad de desarrollar nuevos tratamientos para el TE.

Estudios recientes indican que el temblor en el TE se origina debido a la proyección de oscilaciones patológicas en las vías cerebelo-tálamo-corticales hacia las motoneuronas que inervan los músculos afectados (Helmich 2013), aunque los mecanismos exactos siguen siendo desconocidos. Una hipótesis clásica propone que la oliva inferior es la causa última de temblor en el TE, debido a oscilaciones anormales en las vías olivo-cerebelosas que se transmiten a los circuitos tálamo-corticales (Deuschl 2000).

Los mecanorreceptores, incluidos los corpúsculos de Paccini y Meissner, son sensibles a los estímulos vibratorios. En animales anestesiados o descerebrados, los corpúsculos de Paccini responden a estímulos de alta frecuencia $(60-600 \mathrm{~Hz})$, mientras que los corpúsculos de Meissner responden a estímulos de frecuencia menor $(10-300 \mathrm{~Hz})$ (Mountcastle 1972). Las respuestas sensoriales de ambos tipos de receptores se proyectan al núcleo cuneiforme ipsilateral (Douglas 1978), el cual, tiene importantes proyecciones al tálamo y a la oliva 
inferior (Douglas 1978; Geborek 2012) y, por lo tanto, puede proporcionar una vía para modular los circuitos que median el temblor en el ET.

El temblor en el ET es tratado principalmente con fármacos o estimulación cerebral profunda, una técnica que requiere neurocirugía (Deuschl 2011). Actualmente, ciertos dispositivos portátiles no invasivos se postulan como alternativas a esta medicación, es el caso de sistemas de estimulación de vías aferentes (Dideriksen 2017; Dosen 2015) como el que nosotros planteamos, pero que, por el contrario, utilizan estimulación eléctrica. Aunque muchos de ellos mostraron mejoras claras durante los ensayos clínicos en la población analizada, ninguno de ellos, hasta donde sabemos, ha ido más allá de los ensayos de laboratorio.

En este documento se pretende analizar si los estímulos aferentes administrados a través de la vibración mecánica de la mano y el antebrazo podrían atenuar el temblor en TE. Nuestra hipótesis es que la vibración mecánica estimularía los corpúsculos de Paccini y, por lo tanto, modularía la actividad anormal en las vías relacionadas con el temblor, lo que a su vez reduciría su amplitud. Sin embargo, nuestros resultados no apoyan dicha hipótesis. Encontramos que en una muestra relativamente grande de pacientes $(n=18)$, la respuesta a la vibración fue en gran parte heterogénea, aumentando, disminuyendo o no afectando la amplitud del temblor, al variar los pacientes y las estrategias de estimulación. Además, también encontramos que durante el desarrollo de ensayos relativamente largos (4 min), la amplitud del temblor no es estable, incluso durante mediciones en ausencia de estimulación.

\section{Materiales y métodos}

\subsection{Plataforma}

Para la validación de la hipótesis antes planteada, se ha diseñado y construido una plataforma que suministra estímulos vibratorios, a diferentes frecuencias, en la mano y antebrazo del usuario, al mismo tiempo que se registra el movimiento de dorsoflexión de la muñeca. Los estímulos vibratorios se administran mediante actuadores piezoeléctricos (modelo QP-10W, para las yemas de los dedos; PPA-4011 para la mano; PPA-1022 para el antebrazo; todos de Mide Technology, EE. UU.), que se controlaron a $5 \mathrm{kHz}$ mediante controladores hápticos piezoeléctricos (DRV8662, Texas Instruments, EE. UU.) a través de una tarjeta de adquisición de datos (NI USB 6003, National Instrument, EE. UU.) conectada a un PC. La amplitud de la vibración mecánica se mantuvo constante durante el experimento y se ajustó a su valor máximo (correspondiendo a voltajes de $50 \mathrm{~V}$ para los PPA-1022, $75 \mathrm{~V}$ para los QP-10W y PPA-4011). El movimiento de la muñeca se monitorizó a $100 \mathrm{~Hz}$ con sensores inerciales (TechMCS, Technaid, SP). 
La estimulación mecánica aferente no reduce sistemáticamente el temblor de pacientes con temblor esencial

\subsection{Pacientes}

Pacientes con temblor esencial (TE) fueron reclutados por el departamento de Neurología del Hospital Universitario 12 de Octubre (Madrid, España). Se incluyeron pacientes mayores de edad que hubieran sido diagnosticados con TE. El comité ético del Hospital 12 de Octubre dio su aprobación al protocolo experimental, garantizando el cumplimiento de la Declaración de Helsinki.

El proceso de reclutamiento finalizó con un total de 18 pacientes aptos para participar en el ensayo, (seis mujeres, doce hombres; edad $75.8 \pm 7.9$ años, media $\pm \mathrm{SD}$; rango, 59-88). La duración promedio de la enfermedad fue de 13.6 \pm 11.2 años (rango 1-40 años). La gravedad del temblor varió de leve a grave, con una puntuación media de $2.1 \pm 0.9$ (rango, 1-4) de acuerdo con la escala de clasificación de temblor de Fahn-Tolosa-Marin.

\subsection{Protocolo experimental}

Los ensayos se llevaron a cabo en el brazo más afectado por el temblor. Durante los experimentos, los actuadores piezoeléctricos se ubicaron sobre las yemas de los dedos, la mano y el antebrazo, las áreas donde la densidad de corpúsculos de Paccini es mayor

a

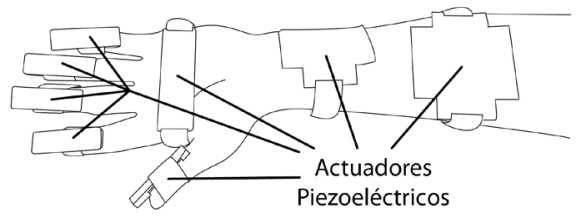

b

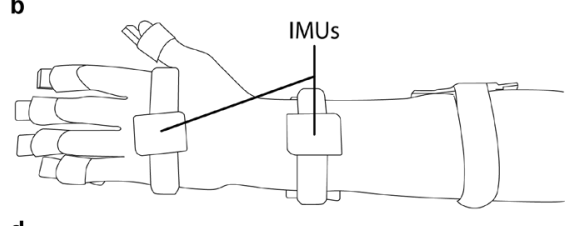

c

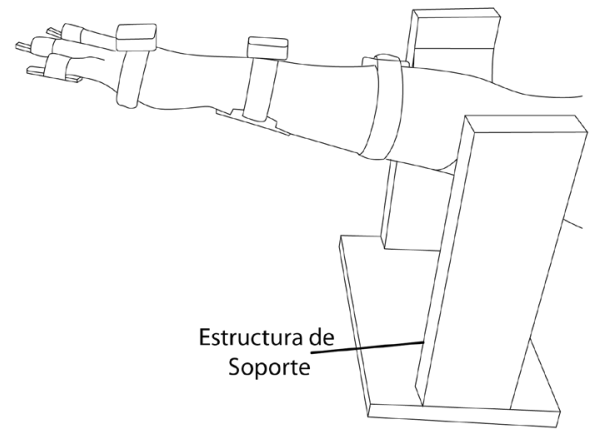

d

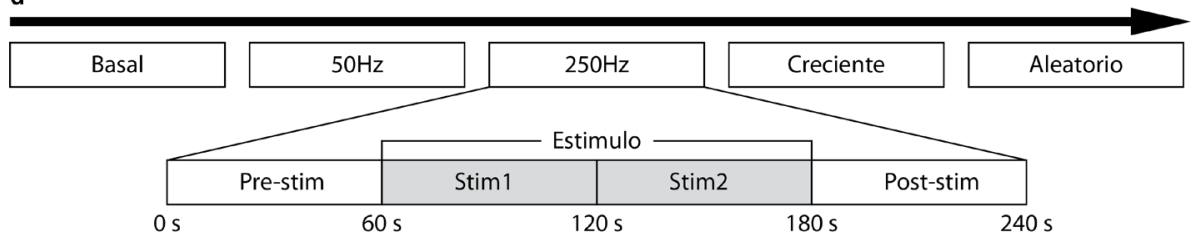

Figura 1. Desarrollo de la sesión y protocolo experimental. a. Localización de los actuadores piezoeléctricos utilizados para aplicar estímulos vibratorios (yemas de los dedos, manos y antebrazos; vista palmar). $b$.

Localización de las IMU utilizadas para registrar el temblor (vista dorsal). c. Estructura de soporte para realizar la tarea postural: los pacientes apoyaron la parte proximal de sus brazos en una banda elástica horizontal. $d$. Esquema del protocolo experimental. En la fila superior, cada rectángulo es una de las cinco estrategias de estimulación; El panel ampliado muestra cómo se dividió cada prueba en cuatro tramos de 60 s. 
(Roudaut 2012) (Figura 1a). Para medir el movimiento de la muñeca, colocamos sensores inerciales (Inertial Measurement Units, IMUs) en la parte dorsal de la mano y el antebrazo (Figura 1b). Los pacientes, sentados frente a un escritorio, realizaron una tarea postural estándar (Figura 1c), mientras que su brazo proximal descansaba sobre un soporte construido para tal propósito (nótese que el soporte no restringe los movimientos de la mano o el antebrazo). El soporte disminuye la fatiga muscular y asegura la repetibilidad en los ensayos. Durante las pruebas, los pacientes recibieron instrucciones de sostener el brazo, el antebrazo y la mano estirados contra la gravedad, para desencadenar el temblor.

El protocolo experimental consistió en cinco ensayos de 4 minutos en los que se aplicaron diferentes estrategias de estimulación (Figura 1d). Entre estos ensayos se intercalaron períodos de descanso de $10 \mathrm{~min}$. A continuación se detallan las citadas estrategias:

1. Sin estimulación ("Basal"): una medida de control en la que se grabó el temblor basal del paciente durante 240 segundos.

2. Estimulación a $50 \mathrm{~Hz}$ (“50Hz”): la vibración se administró a $50 \mathrm{~Hz}$, frecuencia que debería excitar mínimamente los corpúsculos de Paccini (Roudaut 2012).

3. Estimulación a $250 \mathrm{~Hz}$ ("250Hz"): la vibración se administró a $250 \mathrm{~Hz}$, frecuencia que debería excitar al máximo los corpúsculos de Paccini (Roudaut 2012).

4. Frecuencia de estimulación creciente ("Creciente"): la vibración se administró en intervalos de $50 \mathrm{~Hz}$ (orden ascendente, de $50 \mathrm{~Hz}$ a $450 \mathrm{~Hz}$ ); cada frecuencia se aplicó durante $13.33 \mathrm{~s}$.

5. Frecuencia de estimulación aleatoria ("Aleatorio"): la vibración se administró a las mismas frecuencias que durante los ensayos "Creciente", pero en orden aleatorio; Cada frecuencia se aplicó, de nuevo, durante 13.33 segundos.

Los ensayos 2 a 5 se dividieron en cuatro tramos de $60 \mathrm{~s}$ : durante el primer tramo (Pre-stim) evaluamos el temblor basal del paciente; durante los tramos 2 y 3 (Stim1 y Stim2), aplicamos estímulos vibratorios como se define en la estrategia correspondiente $(50 \mathrm{~Hz}, 250 \mathrm{~Hz}$, Creciente, Aleatorio); Durante el último tramo (Post-stim), evaluamos el temblor para detectar posibles efectos retardados.

\subsection{Análisis de los datos}

El temblor de los pacientes fue caracterizado por el movimiento de flexo-extensión de la muñeca, calculándose como la diferencia entre las velocidades angulares del antebrazo y de la mano medidas por los sensores inerciales. Dicha velocidad fue posteriormente filtrada utilizando un filtro paso-banda $\left(10^{\circ}\right.$ orden Butterworth, $\left.\mathrm{fc}=3-12 \mathrm{~Hz}\right)$. Por último, los datos son analizados mediante el valor cuadrático medio (root mean squeared, $R M S$ ) calculado en ventanas no superpuestas de 1 segundo. 
La estimulación mecánica aferente no reduce sistemáticamente el temblor de pacientes con temblor esencial

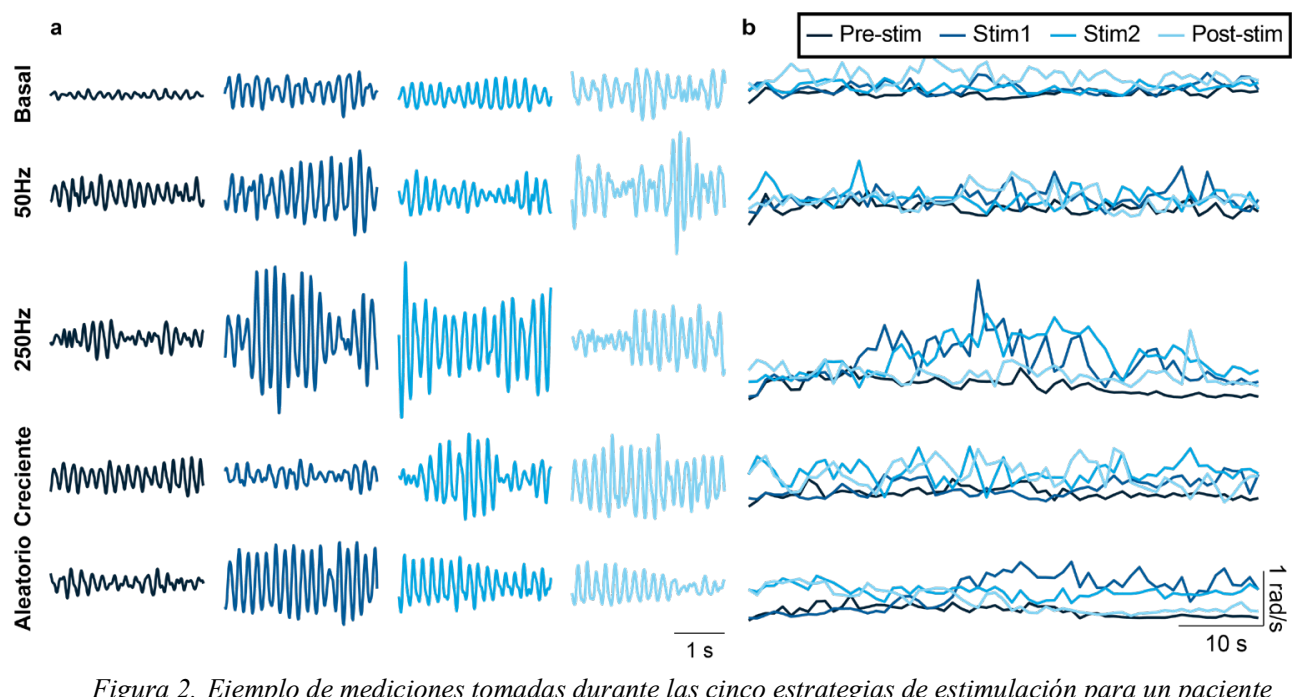

Figura 2. Ejemplo de mediciones tomadas durante las cinco estrategias de estimulación para un paciente (P4). Panel a. Amplitud del temblor (3s de datos) durante cada una de los cuatro tramos (Pre-stim, Stim1, Stim2, Post-stim; mostradas en diferentes columnas) para todas las estrategias de estimulación (agrupadas por fila);

Panel b. Amplitud del temblor durante cada una de las cinco estrategias en el mismo paciente. Cada trazo representa el valor RMS de la amplitud del temblor calculada en ventanas de 1 segundo.

\section{Resultados}

La Figura 2 muestra un ejemplo de los datos obtenidos durante las cinco estrategias de estimulación en un paciente. Como se puede ver en la fila superior de la Figura 2a, la amplitud del temblor varió con el tiempo incluso en la estrategia Basal. La Figura 2b muestra el procesamiento de los datos realizados: estimamos la amplitud del temblor en ventanas de $1 \mathrm{~s}$ no superpuestas para cada una de los tramos de 1 minuto (Pre-stim, Stim1, Stim2, Post-stim).

Nuestro objetivo es analizar la respuesta en cada paciente ante la estimulación, la Figura 3 muestra comparaciones detalladas entre los tramos PreStim y Stim (considerada como la unión de los tramos Stim1 y Stim2) para cada estrategia de estimulación en tres pacientes. En general, el efecto de cada estrategia de estimulación difiere entre ellos. Por ejemplo, durante la estimulación a $250 \mathrm{~Hz}$ : para el paciente P4, la amplitud del temblor aumentó considerablemente (Figura 3g); para el paciente P9, la amplitud del temblor disminuyó considerablemente (Figura 3i); y para el paciente P15, la amplitud del temblor apenas cambió (Figura 3j). Así mismo, incluso cuando no se produce estimulación, el comportamiento es diferente, aumentado, manteniéndose constante o disminuyendo el temblor conforme avanza el tiempo (Figuras 3a, 3b y 3c respectivamente). Esta variabilidad propia del temblor cuando no existe estimulación complica buscar relaciones de causa-efecto, pues no es posible saber si las variaciones que tienen lugar se deben al paso del tiempo o efectivamente son causadas 
por la estimulación. Por este motivo se ha descartado realizar ensayos utilizando los datos medidos después de la estimulación (tramo post-Stim).
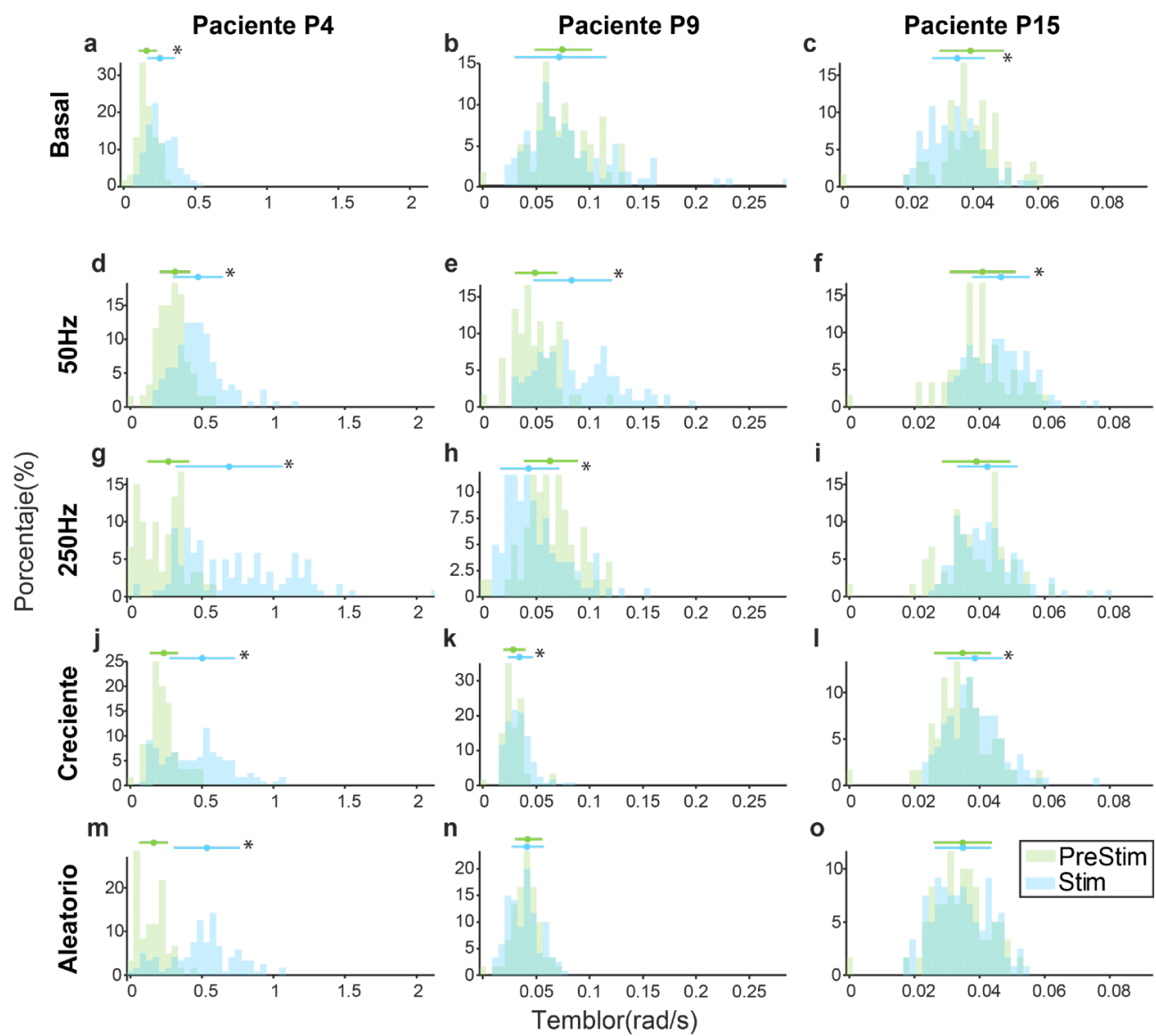

Figura 3. Ejemplos del efecto de las estrategias de estimulación en el temblor de tres pacientes (P4, P9 y P15). Cada panel compara el tramo Pre-stim (verde) con su correspondiente tramo de estimulación (Stim1 + Stim2; en azul). Cada histograma representa la distribución de la amplitud del temblor en intervalos de 1 s para la condición de estimulación correspondiente. Barras superiores: media $\pm S D$; * indica que los tramos Pre-Stim y Stim son significativamente diferentes $(P<0.01$, Test Mann-Witney).

La Figura 4 resume este análisis para los 18 pacientes. En general, todas las estrategias de estimulación aumentaron la amplitud del temblor en la mayoría de los casos, indicándose como la porción dominante en rojo en los resultados agrupados por estrategia estimulación (Figura 4b). Sin embargo, también hubo algunos pacientes para quienes el temblor se redujo o no se vio afectado (Figura 4a). Esta misma respuesta heterogénea se observó durante los ensayos NoStim. Además, incluso para los seis pacientes cuyo temblor se mantuvo sin 

esencial

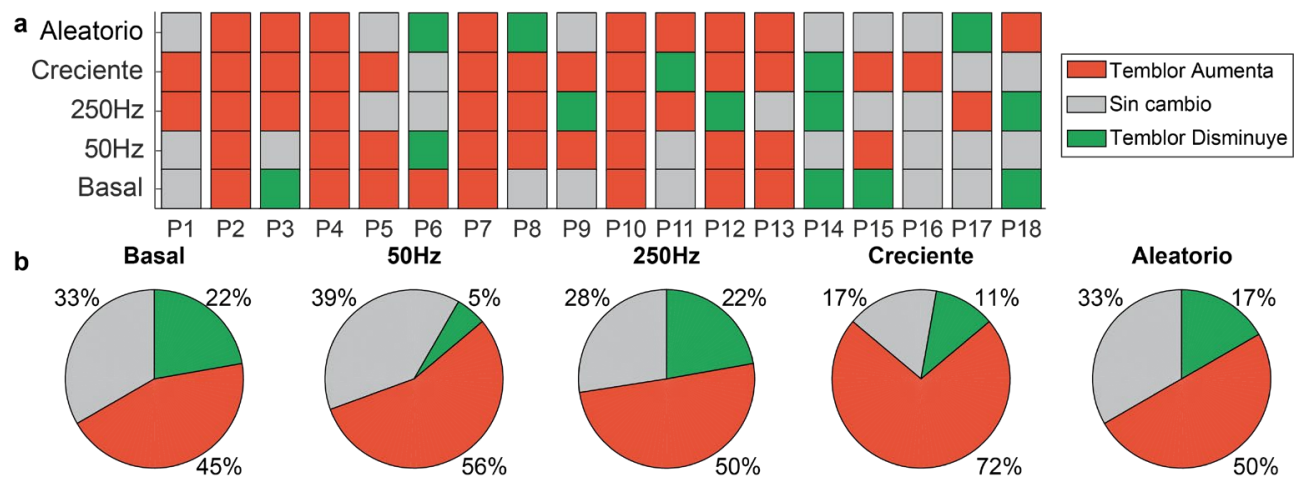

Figura 4. Resumen de la evolución en la amplitud del temblor durante las diferentes estrategias de estimulación. Panel a. Cambio en la amplitud del temblor durante cada tramo Stim con respecto al correspondiente tramo Pre-stim en cada paciente y cada estrategia de estimulación. Panel b. Porcentaje de pacientes para los cuales el temblor disminuyó, aumentó o permaneció inalterado.

cambios durante el ensayo NoStim, los cambios en la amplitud del temblor durante los ensayos de estimulación fueron muy heterogéneos, lo que resultó en un temblor que se incrementó principalmente ( $46 \%$; teniendo en cuenta todas las estrategias de estimulación en todos los pacientes), pero también disminuyeron (17\%) o no se vieron afectados $(37 \%)$.

Debido a la tendencia global, según la cual parece que el temblor aumentase al aplicar la estimulación, se ha intentado identificar un efecto de grupo, normalizando la amplitud del temblor dentro de cada paciente a su percentil 99 y agrupando los datos. El análisis de los resultados de estos grupos reveló un aumento significativo del temblor para todas las estrategias de estimulación ( $\mathrm{P} \sim 0$ para todas las comparaciones; Wilcoxon Rank Sum test; Figura 5), incluso en los ensayos NoStim.

\section{Discusión}

En este trabajo se ha analizado el efecto de la vibración mecánica del antebrazo y la mano sobre el temblor en TE. Nuestro objetivo era estimular selectivamente los corpúsculos de Paccini para interrumpir los mecanismos de generación del temblor en el cerebro, atenuándolo como consecuencia. Para ese fin, diseñamos un protocolo en el que suministramos estimulación vibratoria a diferente frecuencia y estudiamos su impacto en el temblor. En general, aunque la tendencia global parece ser que la vibración mecánica supone un aumento en la amplitud del temblor, la variabilidad entre pacientes fue excesiva como para sacar conclusiones significativas. 


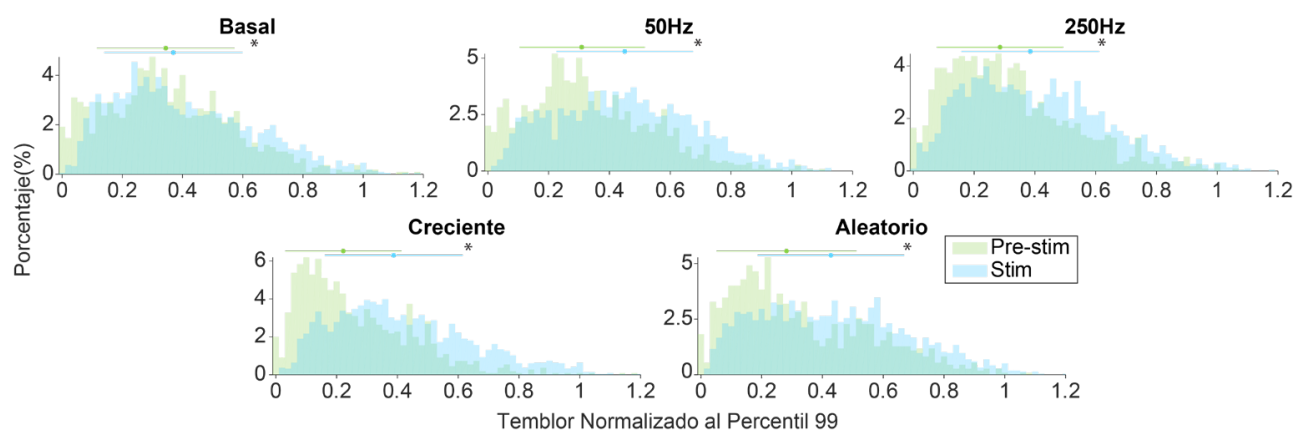

Figura 5. Análisis grupal de la evolución en la amplitud del temblor durante cada estrategia de estimulación. Cada panel compara la amplitud del temblor durante una estrategia de estimulación (azul) con su amplitud durante el tramo de pre-estimulación (verde). Para cada paciente y estrategia de estimulación, normalizamos la amplitud del temblor dividiéndolo por el percentil 99 de su distribución. Barras superiores: media $\pm S D$; * denota que los tramos Pre-Stim y Stim son significativamente diferentes $(P<0.01$, prueba de Wilcoxon Rank Sum test)

Hasta donde sabemos, ningún estudio previo había investigado el efecto de la vibración mecánica sobre el temblor en TE. Sin embargo, algunos grupos han usado la estimulación eléctrica sensorial (por debajo del umbral del motor) para tratar de reducir el temblor en el ET. En (Heo 2015), los autores demostraron que la estimulación eléctrica de los músculos principales del brazo y la muñeca a $100 \mathrm{~Hz}$ reduce la amplitud del temblor en TE. A pesar de que los resultados de su grupo fueron estadísticamente significativos, sus datos también parecen ser muy variables entre los pacientes y los ensayos, dada la amplitud de sus distribuciones de datos. Dideriksen et al. utilizaron recientemente la estimulación intramuscular y de superficie, también administrada a $100 \mathrm{~Hz}$, para estimular las fibras aferentes (entre otras vías) y reducir el temblor de cuatro pacientes con ET (Dideriksen 2017). Por lo tanto, la vibración mecánica parece afectar el temblor de una manera fundamentalmente diferente a la estimulación eléctrica aferente, pues la tendencia vista en nuestros ensayos es que el temblor se incrementaba.

\section{Conclusión}

En este documento se ha presenta el desarrollo de un sistema para reducir el temblor en el Temblor Esencial mediante la aplicación de estímulos vibratorios en el antebrazo y la mano. Nuestros resultados indican que la vibración mecánica estaba interrelacionada con cambios en gran parte heterogéneos en el temblor de los pacientes, aunque la tendencia dominante es a incrementar la amplitud del temblor. Cabe destacar que, durante nuestros ensayos 
La estimulación mecánica aferente no reduce sistemáticamente el temblor de pacientes con temblor esencial

relativamente largos, la variabilidad intrínseca del temblor, incluso sin estimulación, fue mayor de lo que esperábamos. Esta observación dificulta aún más la interpretación de nuestros datos, pero también sugiere que los nuevos protocolos experimentales deberían tener en cuenta la variabilidad intrínseca del temblor a la hora de analizar los resultados.

\section{Referencias}

Deuschl, Günther, Peter Bain, Mitchell Brin, Y Agid, L Benabid, R Benecke, A Berardelli, ET AL. 1998. "Consensus Statement of the Movement Disorder Society on Tremor.” Mov. Disord. 13 (S3): 2-23. https://doi.org/10.1002/mds.870131303.

Deuschl, GÜNTher, AND Rodger ElBle. 2000. "The Pathophysiology of Essential Tremor." Neurology $54 \quad$ (11 $\quad$ Suppl 4): http://www.ncbi.nlm.nih.gov/pubmed/10854347.

Deuschl, Günther, Jan Raethjen, Helge Hellriegel, AND Rodger Elble. 2011. Treatment of Patients with Essential Tremor. The Lancet Neurology. Vol. 10. https://doi.org/10.1016/S1474-4422(10)70322-7.

Dideriksen, Jakob L, Christopher M Laine, Strahinja Dosen, Silvia Muceli, Eduardo Rocon, José L Pons, Julian Benito-Leon, and Dario Farina. 2017. "Electrical Stimulation of Afferent Pathways for the Suppression of Pathological Tremor." Frontiers in Neuroscience 11 . https://doi.org/10.3389/fnins.2017.00178.

Dosen, Strahinja, Silvia Muceli, Jakob Lund Dideriksen, Juan Pablo Romero, EDUARDO ROCON, JoSE PONS, AND DARIO FARINA. 2015. “Online Tremor Suppression Using Electromyography and Low-Level Electrical Stimulation.” IEEE Transactions on Neural Systems and Rehabilitation Engineering 23 (3): 385-95. https://doi.org/10.1109/TNSRE.2014.2328296.

DOUGLAS, P R, D G FERringTON, AND M Rowe. 1978. "Coding of Information about Tactile Stimuli by Neurones of the Cuneate Nucleus." The Journal of Physiology 285 (1): 493513. https://doi.org/10.1113/jphysiol.1978.sp012585.

GEBOREK, PONTUS, HENRIK JÖRNTELl, AND FREDRIK BENGTSSON. 2012. "Stimulation within the Cuneate Nucleus Suppresses Synaptic Activation of Climbing Fibers." Frontiers in Neural Circuits 6 (January): 120. https://doi.org/10.3389/fncir.2012.00120.

Helmich, Rick C., IVAN TONi, GÜNTHER DEUSChl, AND BASTIAAN R. BloEM. 2013. "The Pathophysiology of Essential Tremor and Parkinson's Tremor." Current Neurology and Neuroscience Reports 13 (9): 378. https://doi.org/10.1007/s11910-013-0378-8.

Heo, Jae-Hoon Hoon, Ji-Won Won Kim, Yuri Kwon, SAnG-Ki Ki LeE, Gwang-Moon Moon Eom, Do-Young Young Kwon, Chan-Nyeong NyeOng LeE, Kun-Woo WOO PARK, AND MARIO MANTO. 2015. "Sensory Electrical Stimulation for Suppression of Postural Tremor in Patients with Essential Tremor." Bio-Medical Materials and Engineering 26: S803-9. https://doi.org/10.3233/BME-151372. 
LOUIS, ElAN D. 2005. "Essential Tremor." The Lancet Neurology 4 (2): 100-110. https://doi.org/10.1016/S1474-4422(05)00991-9.

MountCastle, V B, R H LAMotTe, AND G CARLI. 1972. "Detection Thresholds for Stimuli in Humans and Monkeys: Comparison with Threshold Events in Mechanoreceptive Afferent Nerve Fibers Innervating the Monkey Hand." Journal of Neurophysiology 35 (1): 122-36. https://doi.org/10.1152/jn.1972.35.1.122.

Roudaut, Yann, Aurélie Lonigro, Bertrand Coste, Jizhe HaO, PATRick Delmas, AND MARCEL CREST. 2012. "Touch Sense: Functional Organization and Molecular Determinants of Mechanosensitive Receptors." Channels (Austin, Tex.). Taylor \& Francis. https://doi.org/10.4161/chan.22213. 